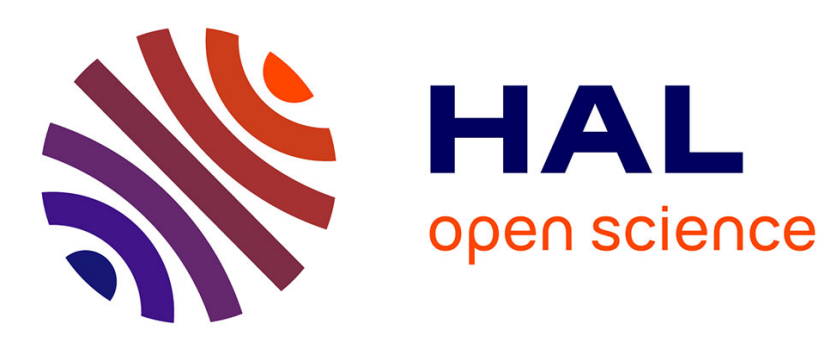

\title{
Jean-Claude Bureau, Sophie Thoyer, 2014, La politique agricole commune, Paris, France, La Découverte, 124 p Eve Fouilleux
}

\section{To cite this version:}

Eve Fouilleux. Jean-Claude Bureau, Sophie Thoyer, 2014, La politique agricole commune, Paris, France, La Découverte, 124 p. Revue d'Etudes en Agriculture et Environnement - Review of agricultural and environmental studies, 2016, pp.755-761. hal-01885275

\section{HAL Id: hal-01885275 \\ https://hal.science/hal-01885275}

Submitted on 10 Oct 2018

HAL is a multi-disciplinary open access archive for the deposit and dissemination of scientific research documents, whether they are published or not. The documents may come from teaching and research institutions in France or abroad, or from public or private research centers.
L'archive ouverte pluridisciplinaire HAL, est destinée au dépôt et à la diffusion de documents scientifiques de niveau recherche, publiés ou non, émanant des établissements d'enseignement et de recherche français ou étrangers, des laboratoires publics ou privés. 


\section{Jean-Claude Bureau, Sophie Thoyer, 2014, La politique agricole commune, Paris, France, La Découverte, 124 p.}

Voilà un ouvrage qui vient à point nommé en ces temps agricoles troublés, présentant de manière à la fois riche et synthétique tout ce que vous avez toujours voulu savoir sur la Politique agricole commune (PAC) sans oser le demander. Assez classiquement, le premier chapitre présente le cadre institutionnel (acteurs institutionnels européens et processus de décision) et les principaux mécanismes sur lesquels s'appuie cette politique (organisations communes de marché, instruments financiers, intervention), ses succès et ses limites au cours du temps, ainsi qu'un panorama des changements introduits par les réformes successives qu'elle a subi depuis les années 1990 (réforme Mac Sharry, Agenda 2000, réformes de 2003 et 2008). Après une présentation générale de l'hétérogénéité des agricultures européennes et une analyse du budget de la $\mathrm{Pac}$, le deuxième chapitre s'attarde plus précisément sur la dernière réforme et les innovations qu'elle a introduites pour la période 2014-2020. Le troisième chapitre passe en revue les instruments et mécanismes économiques sur lesquels s'appuie - ou s'est appuyée - cette politique (prix garantis, quotas, subventions à l'exportation, gel des terres, aides directes, découplage) et les débats plus généraux qui leur sont corrélés (plus ou moins d'intervention étatique ?, Des prix « rémunérateurs » qu'est-ce que ça veut dire ?, Privilégier les petites exploitations durables ?, Quels effets redistributifs entre agriculteurs ?, Quels effets sur les consommateurs ?, etc.). Le quatrième chapitre propose une réflexion sur la PAC et les aspects multifonctionnels de l'agriculture, en évoquant successivement les enjeux d'indépendance alimentaire et de souveraineté nationale, l'aménagement du territoire, la qualité de l'alimentation et les externalités environnementales de l'agriculture, tant négatives que positives. En lien avec ce dernier point, les auteurs font ensuite une revue très claire des différents instruments en place (réglementation, taxations, mesures incitatives) et de leurs impacts potentiels. Le cinquième chapitre porte sur la PAC et les pays tiers; il aborde cette question selon une grille de lecture d'économie internationale, qui permet notamment aux auteurs de déconstruire en grande partie l'accusation de protectionnisme agricole de l'Union européenne. Il évoque les principaux accords et négociations internationales passés, en cours et à venir, tant multi(GATT, OMC) que bi-latéraux (TTIP) et les contraintes qu'ils font peser sur la PAC.

Cet ouvrage est une réédition de celui de Jean-Christophe Bureau (professeur d'économie agricole à AgroParisTech) de 2007, à laquelle Sophie Thoyer (professeure d'économie agricole à Montpellier SupAgro) est venue apporter son heureux concours. Cette nouvelle version est enrichie par de nombreux encadrés et par la description des dernières réformes de la PAC (celle de 2008 et plus particulièrement celle de 2014). On apprécie aussi tout particulièrement le choix de mettre systématiquement les propositions analytiques et illustrations graphiques issues de la théorie microéconomique (mécanismes d'intervention, effets internationaux des instruments, variations 
de surplus...) dans des encadrés spécifiques. Cette réédition est aussi plus étoffée, plus fine, notamment dans son analyse des enjeux environnementaux liés à la PAC, avec sur ce sujet des références souvent plus récentes et plus à jour que sur d'autres aspects traités dans l'ouvrage. Peut-être la co-écriture, qui est indéniablement un plus en l'occurrence, explique-t-elle néanmoins une certaine hésitation normative entre d'une part une approche considérant la «compétitivité dégradée de l'agriculture » comme « la question de fond » (page 95), l'innovation technologique comme facteur de performances accrue (exemple de la production porcine au Danemark page 35) et la question de la compétitivité sur les marchés internationaux comme un impératif, et d'autre part une approche insistant sur les problèmes environnementaux liés à l'agriculture, la qualité de l'alimentation, la question du lien au territoire et à la production des paysages, l'émergence d'attentes nouvelles des citoyens, etc. qui font peser de toutes autres exigences sur l'agriculture et la politique agricole. On note aussi des effets de présentation parfois étonnants comme par exemple au sujet du soutien aux petites exploitations agroécologiques page 69, avec cette mise en garde au nom des économistes selon laquelle « les marchés étant liés, il faut aussi s'assurer que les effets indirects des pratiques écologiques [... ] n'entraînent pas des externalités négatives dans d'autres pays ou secteurs par des changements d'affectation indirecte d'usage des sols», sans pour autant souligner que les productions intensives contemporaines, qu'elles soient porcines, avicoles ou laitières, et qu'elles soient bretonnes, danoises ou espagnoles, s'appuient depuis des décennies sur des changements massifs d'affectation de l'usage des sols en Amérique Latine, où la culture du soja est directement responsable de la déforestation, et de la dégradation de l'environnement et de la qualité des sols et des eaux sur d'immenses surfaces. Quels gains, quelles pertes dans d'autres pays en cas de substitution pour des pratiques agroécologiques en Europe, donc?

Comme ses auteurs l'annoncent d'emblée en introduction, l'ouvrage propose une lecture de la politique agricole commune par la science économique exclusivement. Si cette perspective est à la fois intéressante et absolument essentielle tant pour comprendre les mécanismes de fonctionnement et les effets redistributifs de cette politique publique que pour déchiffrer les controverses au sujet de ses instruments (qui sont principalement des instruments économiques, même si différents aspects réglementaires sont aussi abordés dans l'ouvrage), la théorie microéconomique ne permet pas de tout expliquer néanmoins, et en particulier elle n'est pas outillée pour analyser le «pourquoi » des politiques, bien qu'elle s'y essaie parfois (ainsi que l'illustrent notamment les pages 55 à 58 ). Les auteurs semblent implicitement le reconnaître d'ailleurs en soulignant que "les arguments économiques ne légitiment pas toutes les actions de l'État » et qu'il est "souvent difficile de savoir, lorsque l'État intervient dans un secteur, s'il agit de manière à remédier à une défaillance du marché collectivement coûteuse ou bien s'il œuvre pour privilégier tel ou tel groupe social au détriment de l'intérêt général ». Ils déplorent en outre le faible nombre de travaux récents abordant 
la PAC dans cette perspective. Mais plutôt que d'envisager ce constat comme une limite ou un manque, j'y vois plutôt une opportunité de dialogue avec les autres disciplines des sciences sociales (droit, sociologie, anthropologie, science politique) qui, à partir de leurs épistémologies propres, peuvent apporter des éléments forts utiles dans ce domaine. C'est ce que j'essaie d'illustrer dans les paragraphes suivants à travers mon regard de politiste. Car c'est précisément à ce type de questions liées au "pourquoi ? " que vise à répondre la science politique quand elle choisit d'analyser les politiques publiques.

Pour répondre à ces questions, il est utile de déployer les méthodes et analyses de la sociologie politique, où les acteurs renvoient à des individus ou à des groupes sociaux dotés de ressources différentes et déployant différents types de stratégies pour légitimer leur place dans l'échange politique et le contenu des propositions qu'ils défendent concernant une politique ou un secteur donné (Smith et Hassenteufel, 2002). Acteurs desquels font partie tant les différentes administrations nationales, infranationales, internationales, agricoles et non agricoles (environnement, finances, santé, etc.), que les différents représentants des agriculteurs, des mouvements sociaux impliqués sur les thématiques rurales, agricoles ou alimentaires, des industriels, ainsi que les experts économistes eux-mêmes. Une telle approche permet de se départir d'acteurs « théoriques » type producteurs/consommateurs/contribuables, qui ne renvoient pas à la réalité socialement construite (les groupes de défense des consommateurs ou des contribuables par exemple sont quasiment absents des débats sur la PAC). Elle permet aussi de dépasser un cadre reposant sur des hypothèses très simplificatrices sur les intérêts, les préférences ou la fonction objectif des acteurs, en reconnaissant que ces derniers sont également porteurs de représentations, de valeurs et de croyances, qui conditionnent la conception qu'ils se font de leur intérêt et de leurs préférences. Par ailleurs les «préférences » des acteurs ne sont pas figées : elles peuvent au contraire fortement évoluer dans le temps en fonction des ressources (financières, discursives, analytiques, politiques, institutionnelles) déployées par les uns et les autres, de leurs stratégies de persuasion et des recompositions induites dans les coalitions et les rapports de force en jeu.

Il s'agit là de prendre à bras-le-corps les conflits de valeurs autour des politiques agricoles et comment ils se traduisent sous la forme d'arrangements institutionnels (Coleman et al., 1996 ; Daugbjerg et Swinbank, 2012). Ainsi on ne peut expliquer les résistances aux réformes de la Pac uniquement par le positionnement et l'intérêt des «producteurs » organisés en «lobby » ou « groupe de pression ». La FNSEA, les Jeunes Agriculteurs, les organisations spécialisées par produit comme l'AGPB par exemple, la Confédération Paysanne, la Coordination Rurale ou la FNAB en France (ou encore le COPA-COGECA, la Via Campesina, le groupe PAC2013, ou IFOAM au niveau européen) ont des représentations de leurs identités et de leurs intérêts forts différents voire antagonistes et des positionnements sur la Pac très divers les uns des autres. De même chez les environnementalistes, différentes approches de l'écologie sont en compétition, allant des plus conservationnistes 
arguant de séparer strictement zones de protection environnementale et activités agricoles, aux plus holistiques et intégratives basées sur des approches agricoles alternatives ; ces approches différentes se traduisent par des propositions d'instruments de politique agricole fort différentes. Ainsi les formes particulières d'intégration des préoccupations environnementales dans la PAC, que décrit bien l'ouvrage, résultent directement de la construction de coalitions spécifiques alliant experts économistes, groupes conservationnistes britanniques et organisation de défense des propriétaires terriens et de leur déploiement de stratégies discursives particulières (Ansaloni, 2015). Un autre type d'acteur important dont les stratégies d'influence concernant la PAC restent encore trop peu analysées sont les grandes entreprises multinationales tant de l'amont que de l'aval de l'agriculture, alors que plusieurs d'entre elles investissent massivement des ressources dans un travail multiple d'organisation des controverses, de production de discours et de construction du sens dans le domaine de l'agriculture et des politiques agricoles, tant autour du mot d'ordre productionniste que des enjeux de durabilité. Tous ces différents acteurs disposent par ailleurs de ressources financières, humaines, analytiques et discursives disparates. À partir d'enjeux de légitimation réciproques, ils entretiennent des relations plus ou moins intenses avec les partis politiques et les différents niveaux de gouvernement (infranationaux, nationaux et européens) et les administrations. Et c'est bien la combinaison complexe de ces interactions et les rapports de force qui en résultent qui expliquent le cours de la Pac depuis l'origine et nombre de ses contradictions internes, que ce soit entre ses différents instruments ou entre discours et incitations réelles produites par la politique.

En effet, plutôt que la formalisation rationnelle d'objectifs clairs et précis, les politiques publiques sont toujours le fruit de compromis et de rapports de force, résultat d'un processus de construction historique qui explique leurs contradictions et tensions intrinsèques. Ainsi, au sujet du rôle des pouvoirs publics dans le secteur de l'agriculture biologique par exemple, plutôt que "développer des certifications pour que le consommateur ne rejette pas les produits de l'agriculture biologique de peur des contrefaçons» (page 56), l'Union européenne a, en réalité, mis en place une réglementation européenne et un système de certification avant tout dans une optique politique de construction du grand marché européen, tant pour les produits que pour les services associés (certification). Et dans plusieurs États membres, dont l'Angleterre par exemple, la réglementation publique en vigueur est au contraire perçue comme moins-disante (autrement dit elle serait elle-même la contre-façon) par rapport aux standards privés existant dont en particulier celui de la Soil Association, qui règne en maître sur le marché bio britannique et la représentation du secteur.

De même, la formulation et la mise en ouvre des politiques publiques renvoient fondamentalement à des enjeux de légitimation des acteurs, dans lesquelles les stratégies discursives prennent une grande part. Elles passent souvent par des doubles jeux, comme s'y sont longtemps adonnés les 
céréaliers de l'AGPB par exemple, adhérant aux discours publics de la FNSEA pour rassembler les troupes, mais négociant en réalité des mesures sans points communs avec ces discours dans les bureaux des ministres parisiens ou la Commission Européenne. De même, si lors des négociations sur le budget européen ou dans leurs positionnements de politique générale, les Britanniques adoptent des positions anti-Pac et anti- "statut spécifique de l'agriculture " comme cela est rappelé par les auteurs - notamment pour des raisons électorales au niveau national, cela ne les empêche pas d'adopter des positionnements très conservateurs dans les négociations agricoles elles-mêmes. Et contrairement à ce qui est mentionné dans l'ouvrage, pendant très longtemps - et sans doute est-ce toujours le cas - ils ont bénéficié d'un retour budgétaire très important sur la PAC en plus du fameux « rabais britannique »; ils furent par exemple le principal allié de la France pour refuser la compensation modulée des aides initialement proposée par la Commission lors de la réforme Mac Sharry (Fouilleux, 2003). Cet exemple illustre aussi que comprendre une politique publique implique de ne pas rester cantonné au secteur sur lequel elle porte mais de saisir la dynamique plus large de hiérarchisation des priorités politiques dans laquelle elle s'inscrit - ce que reflètent également les liens étroits entre réforme de la PAC et négociations commerciales multilatérales où les arbitrages intersectoriels sont une constante.

Enfin, une autre interface explorée par la science politique est celui entre policy (politique publique) et politics (le jeu des partis politiques, les dynamiques électorales, le débat public, etc.). Il s'agit là bien sûr d'aller au-delà de simples incursions allusives dans le champ du politique, tentation à laquelle les auteurs cèdent au moins une fois dans l'ouvrage en mentionnant sans plus d'explication que "l'idée que l'agriculture mérite un statut spécial rapproche l'extrême droite et l'extrême gauche " (page 51). Ce faisant, ils mettent non seulement dans l'ombre qu'un tel statut a été surtout défendu en ces termes pendant des décennies sur la scène internationale (GATT, OMC) par des gouvernements de droite et de gauche qu'on peut difficilement taxer " d'extrêmes ", mais également le fait que derrière un même terme se cache en réalité une pluralité de projets politiques, qui conferrent à l'agriculture, au rural, et à la technologie des rôles et des places les plus divers dans la société (on pourrait faire le même parallèle avec le terme d'agriculture «durable » pour la période actuelle). L'enjeu est alors à la fois de comprendre la nature de ces projets politiques en concurrence, les processus de légitimation en jeu et les formes de politisation auxquelles ils donnent lieu. Si les travaux sur l'interface policy/politics sont malheureusement peu nombreux concernant la PAC en dehors des études à visée nationale, peut-être se développeront-ils dans l'avenir dans la mesure où le Parlement est devenu « co-décideur » avec le Conseil depuis le Traité de Lisbonne, alors qu'il était antérieurement seulement consulté sur les questions agricoles (hormis pour le vote du budget). Malheureusement, comme cela est expliqué dans l'ouvrage, la réforme de 2014, qui s'est déroulée en régime de co-décision, 
semble pour l'instant avoir plutôt accentué le verrouillage de la Pac plutôt que sa transformation vertueuse. Le Conseil et Parlement ont fait jeu commun pour repousser les avancées proposées par la Commission, tant sur les questions environnementales que sur la modulation : ainsi les paiements verts ont été quasiment vidés de leur substance et le transfert de fonds du second pilier (davantage orienté vers le développement rural et l'agroenvironnement) vers le premier pilier (aides directes héritées du soutien au marché) a été autorisé, contrairement à la tendance affirmée depuis 15 ans qui visait à renforcer progressivement le second au détriment du premier. Si l'on en juge, en tous cas par les résultats de la large consultation opérée par la Commission avant la réforme (corroborés dans plusieurs sondages Eurobaromètre), qui concluaient clairement à une demande citoyenne pour une PAC plus juste et plus respectueuse de l'environnement, une avancée démocratique sur le plan formel dans le système politique européen s'est clairement traduite par une politique publique s'éloignant des attentes des citoyens (Greer, 2015).

Si la rédaction d'une note de lecture force en quelque sorte à un travail d'analyse critique, celle que je viens d'esquisser visait aussi à proposer des approches complémentaires et elle ne doit en aucun cas faire oublier la grande qualité de cet ouvrage sur la PAC, d'analyse économique mais également de synthèse, écrit par deux spécialistes du sujet. Ce livre est donc à mettre entre toutes les mains, tant celles d'étudiants en économie, sociologie ou science politique s'intéressant aux questions agricoles, que celles de chercheurs, journalistes, voire celles de simples citoyens désirant mieux comprendre les enjeux complexes de la politique agricole européenne.

\author{
Ève FOUILLEUX \\ Université de Montpellier-Cepel, CIRAD-Moisa \\ eve.fouilleux@cirad.fr
}

\title{
Bibliographie
}

Ansaloni M. (2015) Le tournant environnemental de la PAC. Débats et coalitions en France, en Hongrie et au Royaume-Uni, Paris, France, L'Harmattan, 372 p.

Coleman W., Skogstad G. et Atkinson M. (1996) Paradigm shifts and policy networks: cumulative change in agriculture, Journal of Public Policy, 16(3), 273-301.

Daugbjerg C et Swinbank A. (2012) An introduction to the "new" politics of agriculture and food, Policy and Society, 31(4), 259-270.

Fouilleux E. (2003) La PAC et ses réformes. Une politique à l'épreuve de la globalisation, Paris, France, L'Harmattan, 403 p. 
Greer A. (2015) Moving beyond de-compartmentalisation? Inter-institutional policy making in agriculture: an examination of the 2013 CAP reform, in: Daugbjerg C and Feindt PH (eds) International Conference on Public Policy, Panel/section T18P02 on The new policy and politics of food and agriculture, 1st-4th July, Milan, Italie, $23 \mathrm{p}$.

Smith A et Hassenteufel P. (2002) Essoufflement ou second souffle ? L'analyse des politiques publiques «à la française ", Revue française de science politique, 52(1), 53-73. 
